\title{
Side Stream Dark Field (SDF) imaging of oral microcirculation in the assessment of systemic sclerosis
}

\author{
M. Sha, M. Griffin $*$, C. Denton, P. Butler
}

Charles Wolfson Center for Reconstructive Surgery, Royal Free Hospital, London, United Kingdom

UCL Centre for Nanotechnology and Regenerative Medicine, Division of Surgery \& Interventional Science, University College London, London, United Kingdom Center for Rheumatology, Royal Free Hospital, London, United Kingdom

Plastic \& Reconstructive Surgery, Royal Free Hospital, London, United Kingdom

\begin{abstract}
Introduction: Systemic sclerosis (SSc) is a systemic disease characterised by abnormalities in small blood vessels, skin and organ fibrosis. It is assessed using generalised skin thickening scores, autoantibodies and nailfold cap- illaroscopy. Side Stream Dark Field imaging (SDF) is a non-invasive imaging tool that assesses microcirculation. This study aims to investigate the potential of using SDF as a diagnostic tool in SSc.
\end{abstract}

Method: Oral microcirculation of 20 patients with SSc was compared to 20 age and gender matched controls us- ing SDF imaging. Sublingual, buccal and incisor regions of the mouth were examined. All volunteers were female averaging 48.0 (24-64) years old. Vasculature was assessed by calculating the De Backer score and Functional Capillary Density (FCD) on an imaging software.

Results: At all regions of the mouth, SSc patients had a significantly lower De Backer score compared to con- trols (SSc $3.484 \pm 0.1361 / \mathrm{mm}$ vs Control $5.184 \pm 0.1896 / \mathrm{mm}$, unpaired t-test $p<0.0001$ ). The SSc patients showed significantly lower FCDs compared to controls at all areas as well (SSc $19.65 \pm$ $0.9445 \%$ vs Control $29.45 \pm 1.681 \%$, unpaired t-test, $p<0.0001$ ). The incisor regions had significantly higher De Backer and FCD scores than buccal and sublingual regions in both control and SSc patients (one way anova, $p<0.05$ ). De Backer/ FCD scores showed significant correlation against Rodnan Skin Scores in patients with SSc (Pearson correlation, $\mathrm{p}<0.05$ ).

Conclusions: SSc patients showed decreased oral vasculature compared to controls. SDF imaging has shown the ability to be a useful diagnostic tool in the assessment of SSc.

Dear Editor,

Systemic sclerosis (SSc) is a rare multisystem autoimmune disease characterised by vasculopathies in small blood vessels, autoantibod- ies and fibrosis of skin and organs. It mostly affects women between 30 and 50 years of age (Walker et al., 2007). Its diagnosis can be aided by the 2013 ACR/EULAR classification criteria: clinical presentations (sclerodactyly, telangiectasia and skin thickening), Raynaud phenome- non, SSc-related autoantibodies and abnormal nailfold capillaries (van den Hoogen et al., 2013). Fibrosis around the mouth results in limited mouth opening in severe cases of SSc. As microvascular dysfunction is regarded an initial pathological event, nailfold 
capillaroscopy has been included in this classification criteria. In these images, cap- illary loops appear dilated (damaged from inflammatory re

actions) and are lost as a result of progressive destruction (Ruaro et al., 2018). Sidestream Dark Field (SDF) imaging is also a non-invasive imag- ing technique that has potential to quantify physiological parameters such as capillary density in mucosal microcirculation.

In this preliminary study we aimed to ascertain the validity of SDF imaging as diagnostic or prognostic markers in assessing SSc. Significant differences in oral capillary density between healthy and SSc patients would lend support to this. Correlation with Rodnan skin scores that assesses systemic skin thickening would also assess the validity of SDF imaging. We also aimed to explore the vascular differences amongst specific sites of the oral mucosa.

In this study, SDF imaging was used to assess the oral microcirculation of 20 patients diagnosed with SSc and compared to 20 age and sex matched controls (Table 1). Assessment was performed at the sub- lingual, buccal and incisor regions. Volunteers were female of an average of 48.0 (24-64) years old. Written informed consent was obtained from each volunteer after a thorough explanation of the study.

Microscopy was performed on each volunteer with a portable video- capillary CapiScope (KK Technology) to view the oral microvasculature. Green light emitting diodes (from the SDF camera) is absorbed by haemoglobin in RBC. This allows RBC visualisation in contrast to the vascular background. The SDF device was equipped with a sterile probe cap and was applied to incisor, buccal and sublingual regions on both sides of the volunteer's mouth. 5-10 s videos were recorded at each site. A tripod was used to minimise movement and pressure artefacts. Salivary secretions were removed with gauze to obtain a clear image.

Using the CapiScope Image acquisition and analysis software, vessel density was assessed by calculating the De Backer score and Functional Capillary Density (FCD) from the video sequences. The De Backer score $(\mathrm{n} / \mathrm{mm} 2)$ was calculated by the number of intersections between the capillaries and a grid of known dimension (De Backer et al., 2007) (Fig. 1). FCD is derived from the image contrast and relies on movement in the vessels such as gaps and white cells in the video sequence. Based on an appropriate threshold, FCD is calculated from the area of functional capillaries in a fixed region of interest.

Statistical analysis was performed using Prism version 7.00. Un- paired t-tests were used to compare differences in De Backer scores and FCDs of each site between healthy controls and SSc patients. A one-way ANOVA was performed to evaluate any difference amongst the 3 cho- sen sites of oral vasculature (incisor, buccal and sublingual). Correlation analysis was performed against the Rodnan skin score obtained from pa- tient records. Statistical significance was set at $p<0.05$ for all analyses. The De Backer score of SSc patients was significantly lower com- pared to controls in all regions of the mouth (SSc $3.484 \pm 0.1361 / \mathrm{mm}$ vs Control $5.184 \pm 0.1896 / \mathrm{mm}$, unpaired t-test $\mathrm{p}<0.0001$ ) (Fig. 2). The FCD of SSC patients was significantly lower compared to controls at all areas (SSc $19.65 \pm$ $0.9445 \%$ vs Control $29.45 \pm 1.681 \%$, unpaired t-test, $p<0.0001$ ). The incisor showed significantly higher De Backer and FCD scores than the buccal and sublingual regions in both control and SSC patients (one-way anova, $p<0.05$ ). There was significant cor- relation between De Backer Score/FCD and Rodnan Skin Score in patients with SSc (Pearson correlation, $\mathrm{p}<0.05$ ). 
This preliminary study reported SSc patients to have significantly de- creased oral microvasculature. In SSc, microvascular damage precedes skin fibrosis and is a key initial marker of disease. The SDF images obtained from this study is consistent with this pathophysiology. Correlation with the established Rodnan Skin scores further strengthens the potential of SDF imaging as a diagnostic tool in SSc. Correlation between nailfold capillaroscopic images and SDF imaging will be investigated in the future.

In conclusion, SDF has been proven to be a valid tool in assessing SSc. There is potential for it to be used in diagnosis and assessing treatment as a prognostic marker.

\section{Funding}

This research did not receive any specific grant from funding agen- cies in the public, commercial, or not-for-profit sectors.

Acknowledgements

None.

References

De Backer, D., Hollenberg, S., Boerma, C., Goedhart, P., Büchele, G., Ospina-Tascon, G., Dobbe, I., Ince, C., 2007. How to evaluate the microcirculation: report of a round table conference. Crit Care 11 (5), R101.

Ruaro, B., Sulli, A., Smith, V., Pizzorni, C., Paolino, S., Alessandri, E., Trombetta, A., Cutolo, M., 2018. Advances in nailfold capillaroscopic analysis in systemic sclerosis. Journal of Scleroderma and Related Disorders 3 (2), 122-131.

van den Hoogen, F., Khanna, D., Fransen, J., Johnson, S.R., Baron, M., Tyndall, A., Matucci-Cerinic, M., Naden, R.P., Medsger, T.A., Carreira, P.E., Riemekasten, G., Clements, P.J., Denton, C.P., Distler, O., Allanore, Y., Furst, D.E., Gabrielli, A., Mayes, M.D., van Laar, J.M., Seibold, J.R., Czirjak, L., Steen, V.D., Inanc, M., Kowal-Bielecka, O., Müller-Ladner, U., Valentini, G., Veale, D.J., Vonk, M.C., Walker, U.A., Chung, L., Collier, D.H., Csuka, M.E., Fessler, B.J., Guiducci, S., Herrick, A., Hsu, V.M., Jimenez, S., Kahaleh, B., Merkel, P.A., Sierakowski, S., Silver, R.M., Simms, R.W., Varga, J., Pope, J.E., 2013. 2013 classification criteria for systemic sclerosis: an American Col- lege of Rheumatology/European League against Rheumatism collaborative initiative. Arthritis Rheum 65 (11), 2737-2747.

Walker, U.A., Tyndall, A., Czirják, L., Denton, C., Farge-Bancel, D., Kowal-Bielecka, O., Müller-Ladner, U., Bocelli-Tyndall, C., Matucci-Cerinic, M., 2007. Clinical risk assess- ment of organ manifestations in systemic sclerosis: a report from the EULAR Sclero- derma Trials And Research group database. Ann Rheum Dis 66 (6), 754-763 
Table 1

Clinical characteristics of the patients identified in the study.

Controls Study patients

Average age (years) $\quad 48 \quad 49$

$\operatorname{Sex}(F: M) \quad 20: 0 \quad 20: 0$

Diffuse scleroderma:limited scleroderma _ _ $\quad$ 4:16

Clinical therapies $\quad-\quad$ Mycophenolate Mofetil: 2 Prednisolone: 5

Methotrexate: 7

Hydroxychloroquine: 15

Iloprost: 10 
Fig. 1. SDF image of a healthy control's left incisor obtained from the CapiScope Image acquisition and analysis software. The 20 intersection points on a fixed grid with known dimensions gives a De Backer score of $20 \div 4.579 \mathrm{~mm}=4.368 / \mathrm{mm}$.

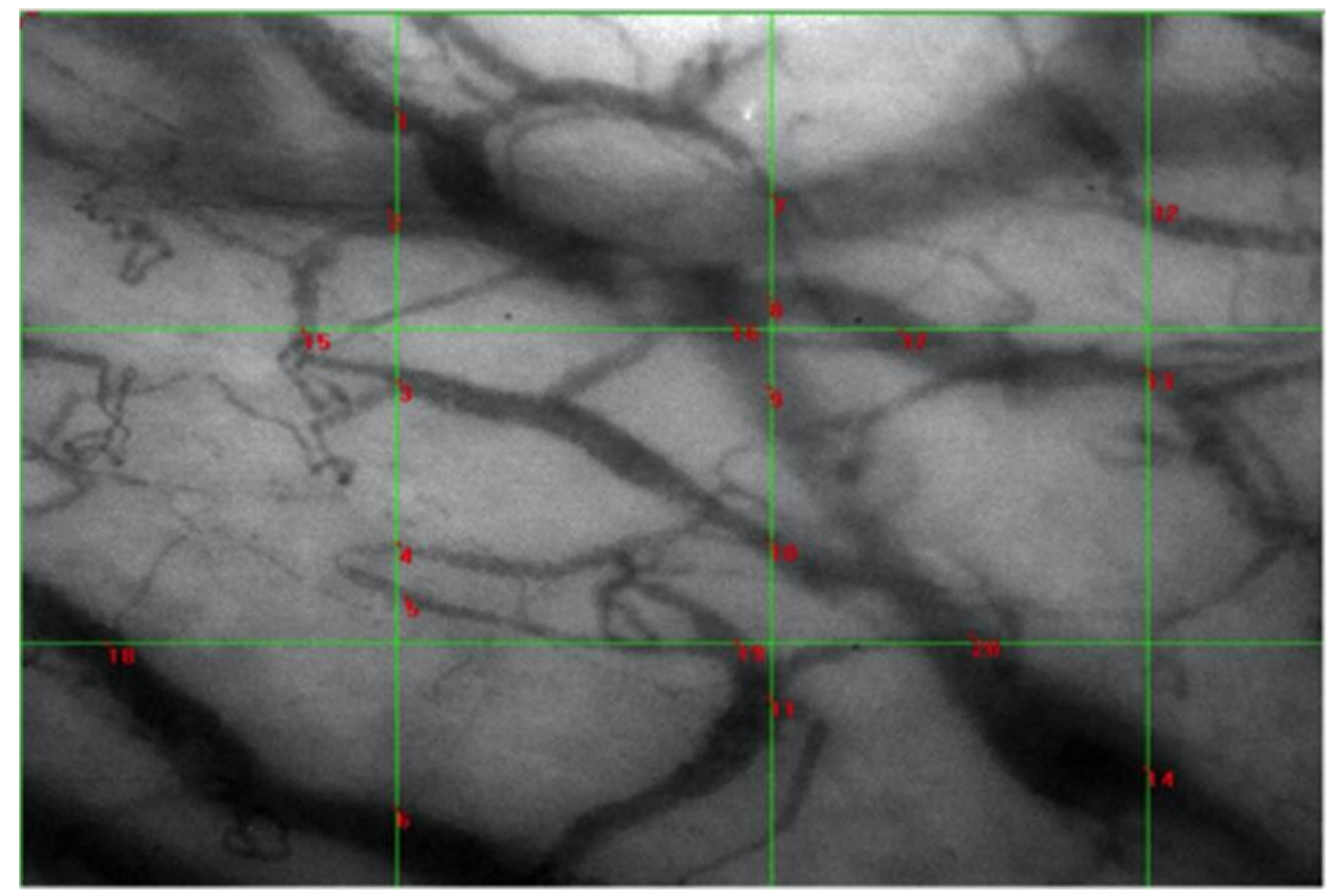


Fig. 2. Comparison of De Backer scores between SSc patients and controls $(N=20)$. The SSC patients had a significantly lower score than patients $(p<0.0001)$ indicating decreased vasculature.

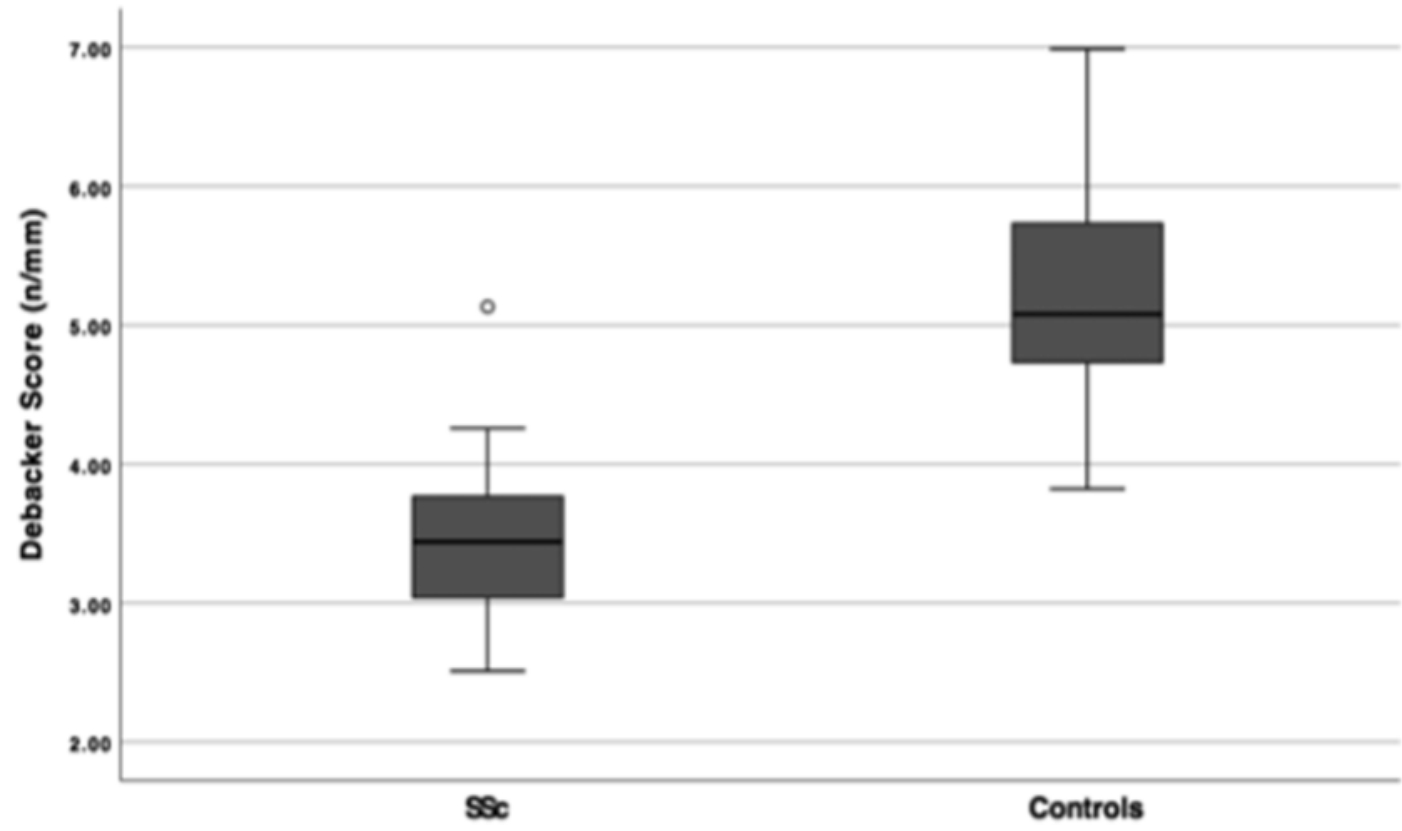

